\title{
Perforansinsuffizienz
}

\section{K. Korsake}

Klinik für Gefäß- und Endovaskuläre Chirurgie/Phlebologie, Städtisches Klinikum Dessau, Dessau-Roßlau

\begin{abstract}
Schlüsselwörter
Perforansvenen, Varikosis, Duplex, Veneninsuffizienz

\section{Zusammenfassung}

Perforansvenen verbinden die epifaszialen Beinvenen mit den subfaszial gelegenen tiefen Venen und dienen zur Drainage des venösen Blutflusses. In der Diagnostik und Therapie einer Varikosis haben die Perforansvenen eine große Bedeutung beim Aufsuchen des proximalen und distalen Insuffizienzpunkt. Die Untersuchung der Perforansvenen ist eine Herausforderung in der Duplexsonographie. Die pathophysiologisch bedeutsamen insuffizienten Perforansvenen sollten operativ oder minimal invasiv ausgeschaltet werden.
\end{abstract}

Korrespondenzadresse

Dr. med. Kristina Korsake

Klinik für Gefäß- und Endovaskuläre Chirurgie/Phlebo-

logie

Städtisches Klinikum Dessau, Auenweg 38

D-06847 Dessau-Roßlau

E-Mail: kristina.korsake@gmail.com

\section{Keywords}

Perforating veins, varikosis, duplex ultrasound, venous insufficiency

\section{Summary}

Perforating veins connect the superficial and deep venous system. In the diagnosis and treatment of varicosis, perforating veins are of high importance in search for the most proximal and most distal point of insufficiency. The examination of the perforating veins is a challenge in duplex sonography. The pathophysiological relevant significant incompetent perforating veins should be treated with surgical or minimal invasive methods.

Perforating veins insufficiency

Phlebologie 2018; 47: 279-285

https://doi.org/10.12687/phleb2442-5-2018

Eingegangen: 10. Juli 2018

Angenommen: 23. Juli 2018

English version available at:

www.thieme.de/phlebo

\section{Definition}

Unter einer Vena perforans versteht man eine Verbindungsvene zwischen einer Vene, die dem tiefen Leitvenensystem zuzurechnen ist, und einer epifaszialen Vene, die die Faszia durchbohrt. Ihre physiologische Flussrichtung ist von der Oberfläche in die Tiefe gerichtet. Bei der Insuffizienz der Perforansvenen, kommt es zu einer Strömungsumkehr in den Perforansvenen, mit Stauungen der oberflächlichen Venen (1) ( Abb. 1).

In der Literatur sind mehr als 40 örtlich konstant vorliegende Perforansvenen beschrieben worden (2). Perforansvenen am Unterschenkel sind wesentlich zahlreicher als am Oberschenkel.
Die am Häufigsten an einer Varikose beteiligten Perforansvenen sind schematisch in $>$ Abbildung 2 und 3 dargestellt.

\section{Klassifikation}

Im klinischen Alltag wurden Perforansvenen oft mit den Namen von Autoren assoziiert. Nach der neueren, internationalen Nomenklatur werden die Perforansvenen nicht mehr durch Eigennamen bezeichnet, sondern auf Basis ihrer Topographie. Die alten Bezeichnungen sind aber im klinischen Sprachgebrauch noch weit verbreitet. (3) Nach der neuen Nomenklatur heißen z.B. die Cockett-Perforantes nun Tibialis posterior Perforansvenen ( Abb. 5 und 6). Einen Überblick zu den alten und neuen Bezeichnungen finden Sie in der $>$ Tabelle 1.

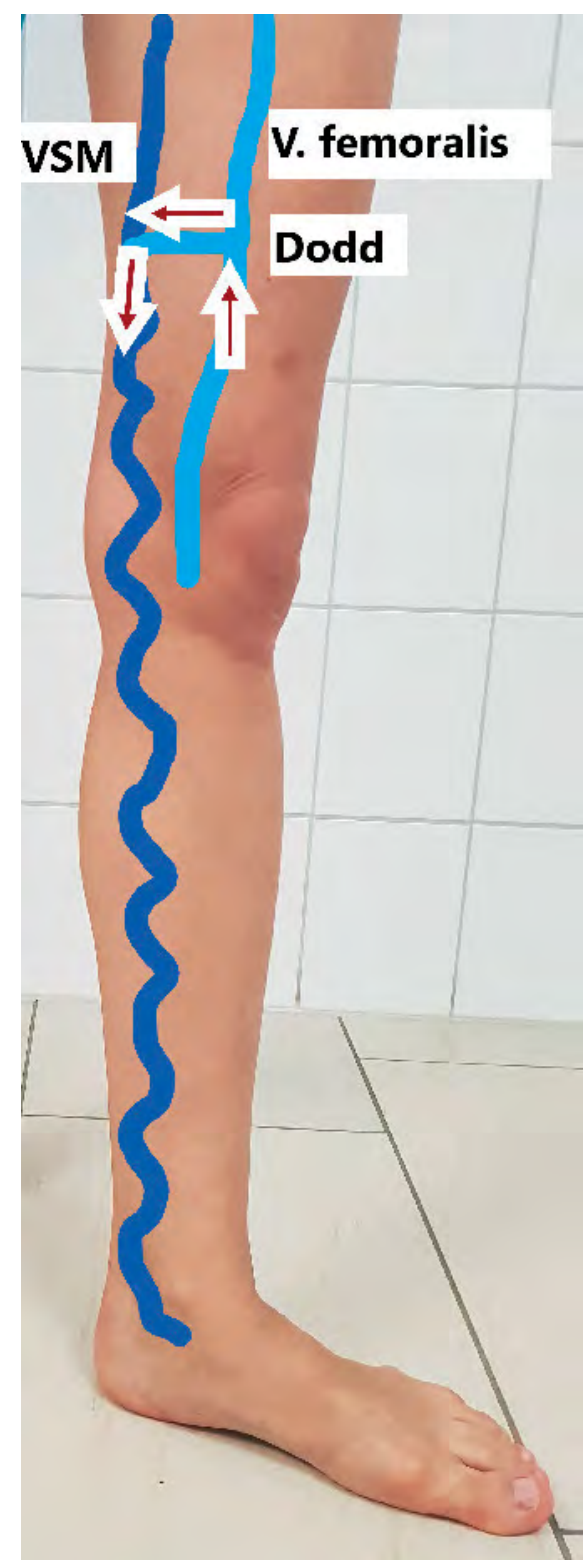

Abb. 1 Insuffiziente Dodd-Perforans mit Flussumkehr in die VSM

\section{Diagnostik}

Im Rahmen der Diagnostik bei Varikosis und CVI kann die Funktion der Perforansvenen durch klinische und bildgebende Verfahren beurteilt werden. 


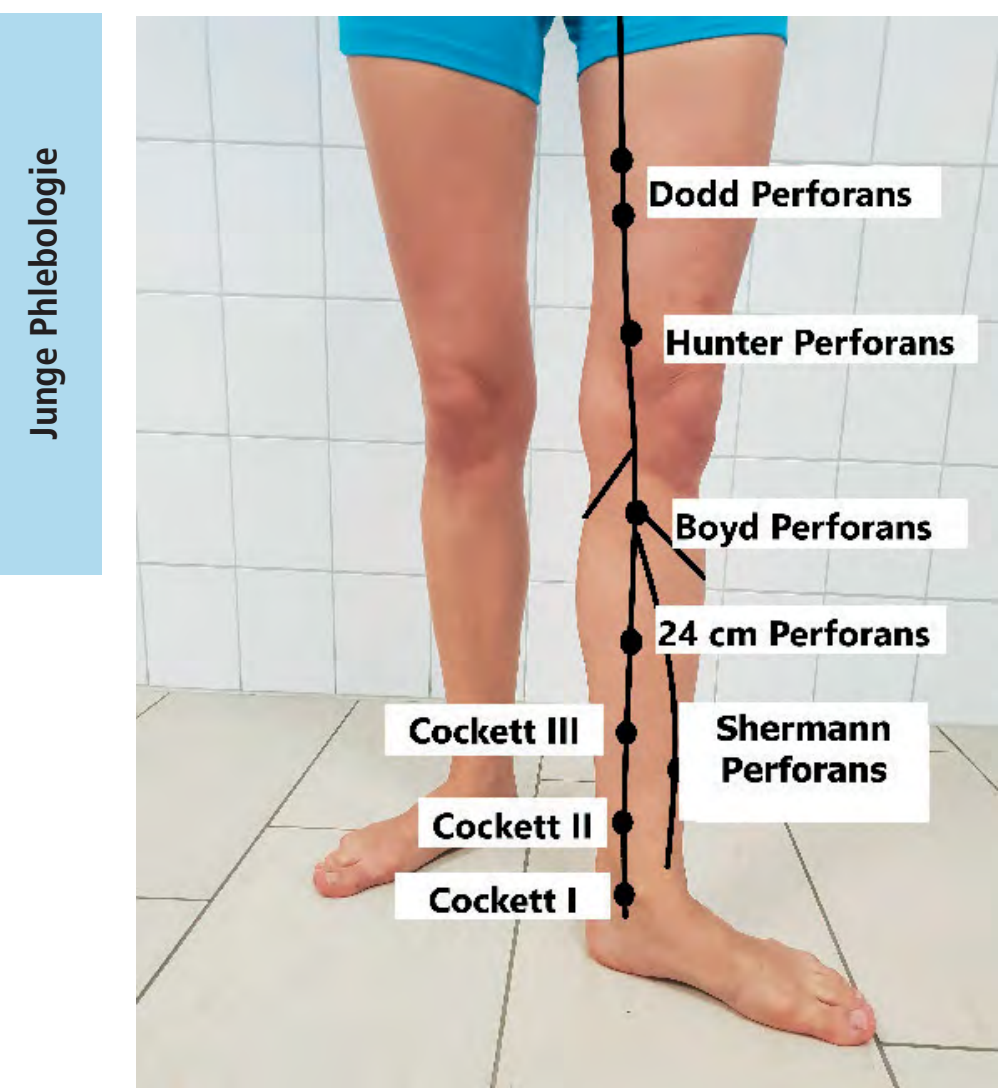

Abb. 2 Lokalisation der bekanntesten Perforansvenen von ventral

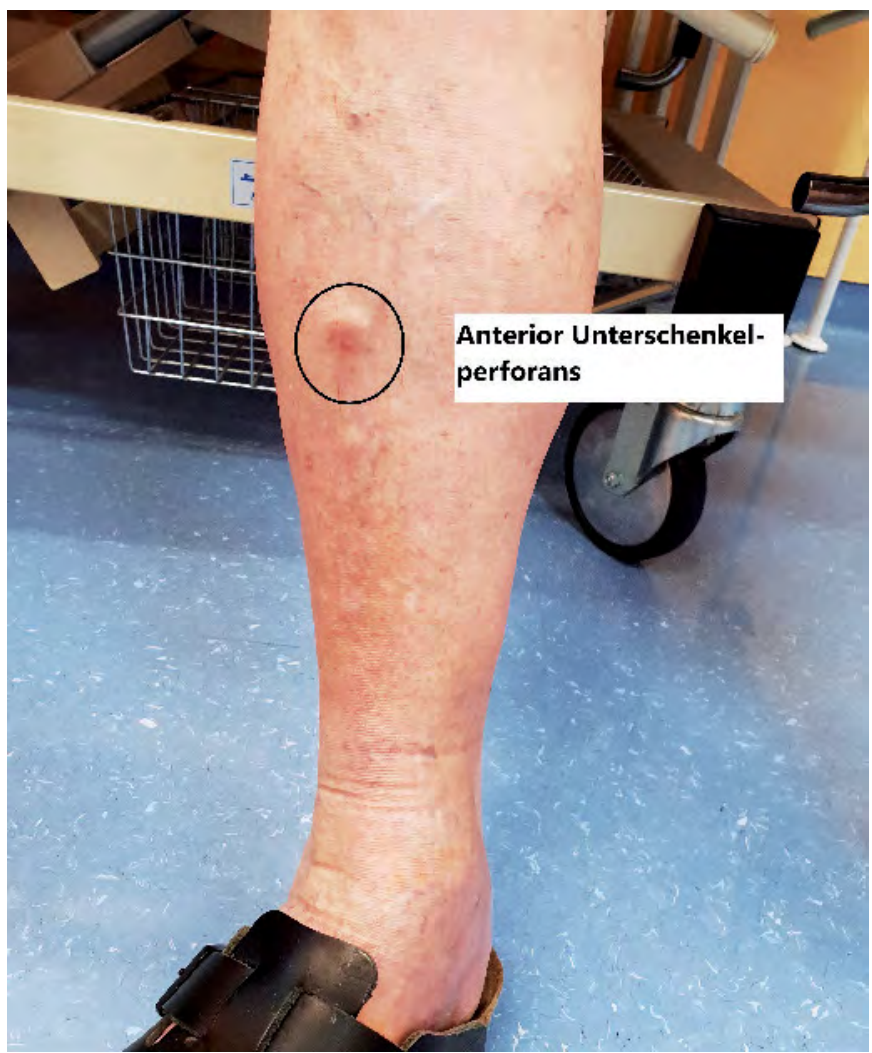

Abb. 4 Paratibiale Perforansvene nach traumatischer Verletzung der Tibia mit typischem Blow-out-Phänomen

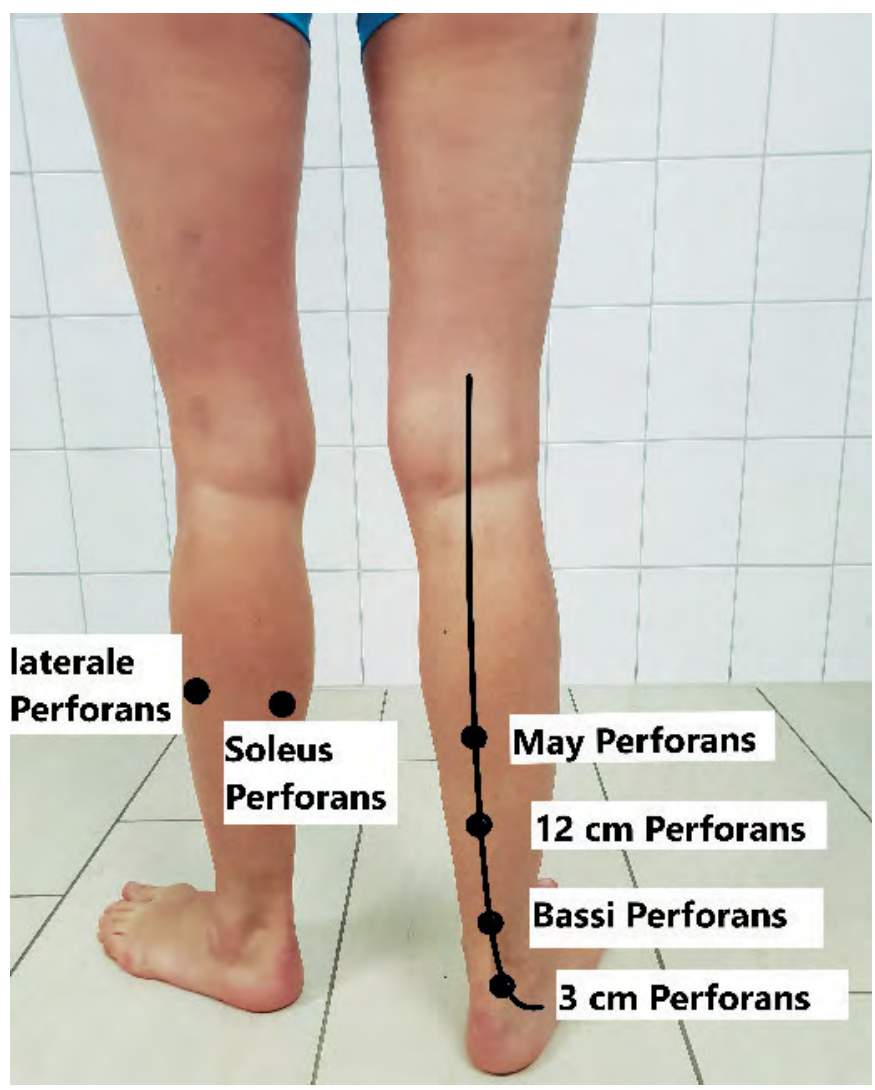

Abb. 3 Lokalisation der Perforansvenen von dorsal

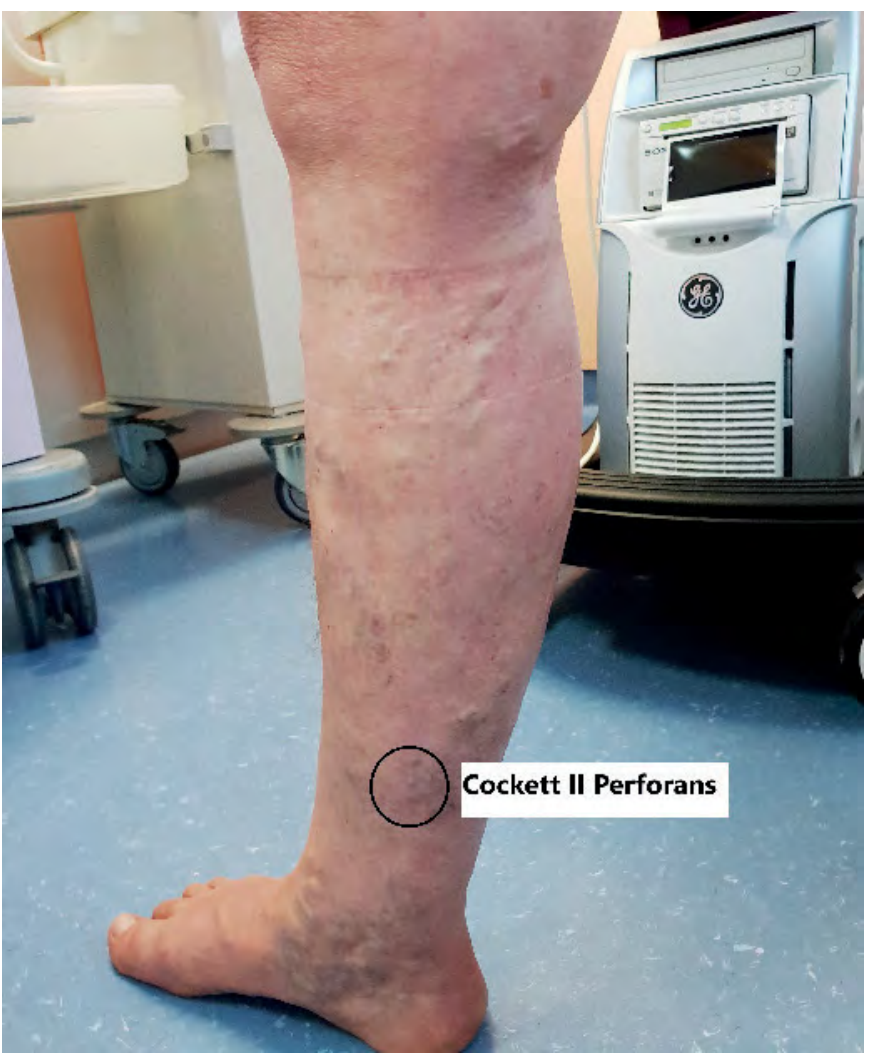

Abb. 5 Rezidivvarikosis nach Crossektomie und Stripping VSM rechts, mit Perforansinsuffizienz Cockett II 


\section{Klinische Diagnostik}

Eine Perforansveneninsuffizienz führt meist zu pathologisch erweiterten Faszienlücken, die bei Palpation häufig schmerzhaft sind. Bereits in der Inspektion des stehenden Patienten fallen insuffiziente Perforansvenen meist durch das sog. Blow-OutPhänomen auf.

Zur weiteren klinischen Diagnostik können Venenfunktiontests (Perthes, Mahorner-Ochsner, Trendelenburger und Pratt-Test) angewendet werden.

\section{Farbkodierte Duplexsono- graphie}

Eine Objektivierung der Perforansinsuffizienz gelingt am einfachsten durch Duplex-Sonographie. Schon im B-Bild erkennt man die erweiterte Perforansvenen $(\triangleright$ Abb. 7).

Im Ultraschall werden meist nur die Perforansvenen gesehen, die pathologisch gedehnt sind. In den meisten fließt das Blut von der Oberfläche in die Tiefe, als Drainage für den Reflux in der Stammvene oder in den Seitenästen zur tiefen Beinvene hin. Bei umgekehrtem Fluss ist eine Perforansvene die Refluxquelle für eine Varikose (4).

Auch in der farbkodierte Duplexsonographie kann man Perforansvenen gut darstellen. Der physiologische Blutfluss in einer Perforans ist von der Oberfläche in die Tiefe gerichtet. Die Flussströmung in einer Perforansvene ist unter dem Valsalva-Provokationsmanöver oder manueller Kompression der Wade darstellbar. So wird der Druck in den tiefen Venen erhöht und das Blut nach proximal und in die Perforansvenen geleitet. Wenn eine Perforansvene aufgrund des Refluxes oder einer Volumenüberlastung erweitert ist, wird während der muskulären Systole ein auswärts gerichteter Fluss vorliegen (4) ( Abb. 8 und 9).

Relevant für die Diagnostik ist der Fluss nach Dekompression. Liegt in der Diastole ein zur Oberfläche gerichteter Fluss vor, ist die Perforansvenen insuffizient.

Die Provokationsmanöver sollten mittels Valsalva- Pressmanöver, Bewegung der Fußspitze (Wuntsdorfer Manöver) oder durch manuelle Wadenkompression erfolgen. Eine proximal der Perforansvene an-

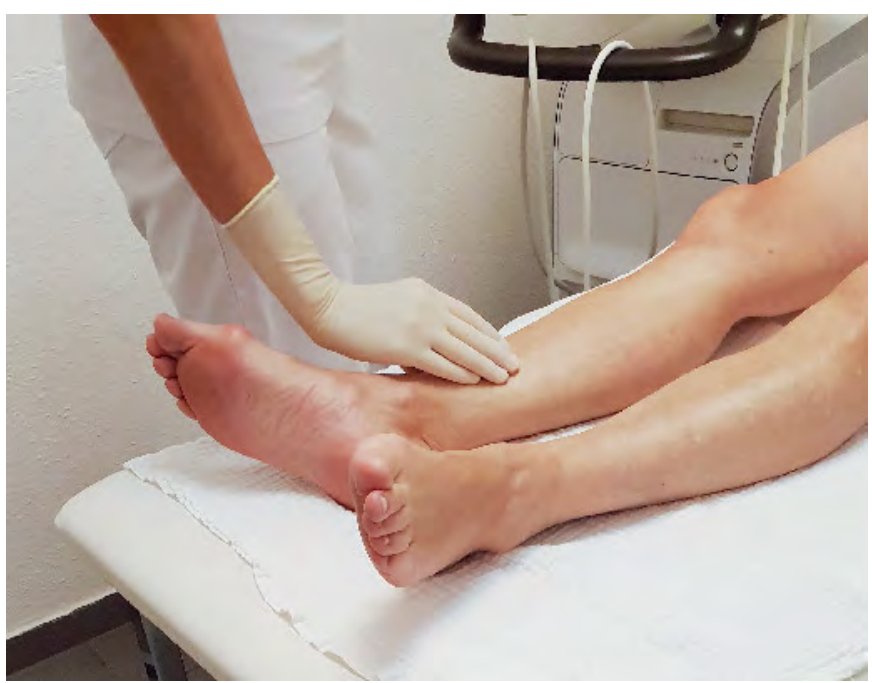

Tab. 1

Topographische Nomenklatur der Perforansvenen

\begin{tabular}{|c|c|}
\hline Alte Nomenklatur & Neue topographische Nomenklatur \\
\hline Cockett-Perforantes (I, II, III) & $\begin{array}{l}\text { Tibialis posterior Perforansvenen (obere, } \\
\text { mittlere und untere) }\end{array}$ \\
\hline Bassi-Perforans & paraachilläre Perforansvenen \\
\hline Shermann Perforans & Paratibiale Perforans \\
\hline $\begin{array}{l}\text { May-Perforans („Mid-calf-Per- } \\
\text { forans") }\end{array}$ & Intergemelläre (soleale) Perforantes \\
\hline Boyd-Perforans & Hintere tibiale Perforans \\
\hline Hunter-Perforans & Inguinale Perforantes \\
\hline Dodd-Perforans & Perforansvenen des Adduktorenkanals \\
\hline Hach-Perforans & Posteriolaterale Perforans \\
\hline
\end{tabular}

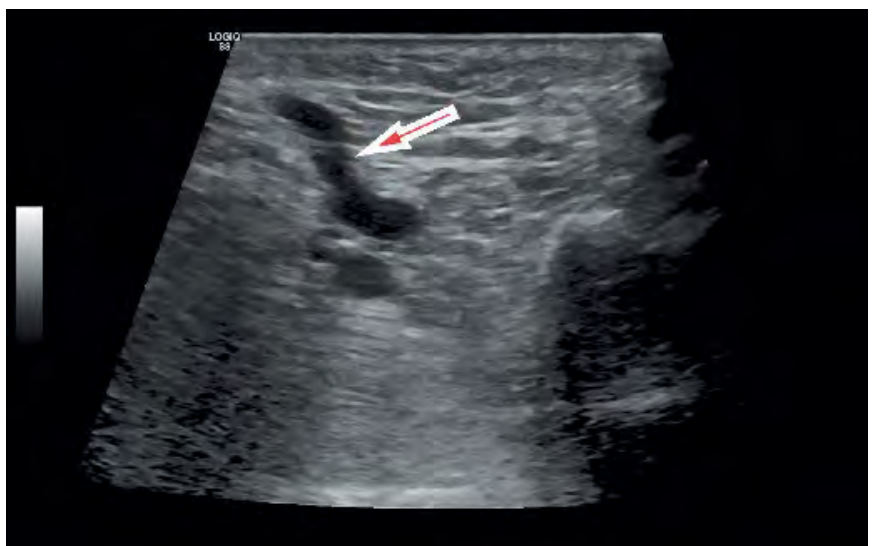

Darstellung einer dilatierte Perforansvene im B-Bild

Palpatorisch erkennt man die insuffiziente Perforansvenen durch erweiterten Faszienlücken

gelegter Stauschlauch beim Manöver kann zu falsch pathologischen Ergebnissen führen, weil durch den Stauschlauch die insuffiziente Vene nach proximal verschlossen und die sonst vorhandene Rezirkulation in der Diastole verhindert wird. Nach der Dekompression wird der Blutfluss aus der tie- fen Venen über die gedehnte Perforansvene in das distale insuffiziente OberflächenSegment abfließen. Somit scheint eine Vene insuffizient, die unter physiologischen Bedingungen ohne Stauschlauch dem Wiedereintritt dient. 


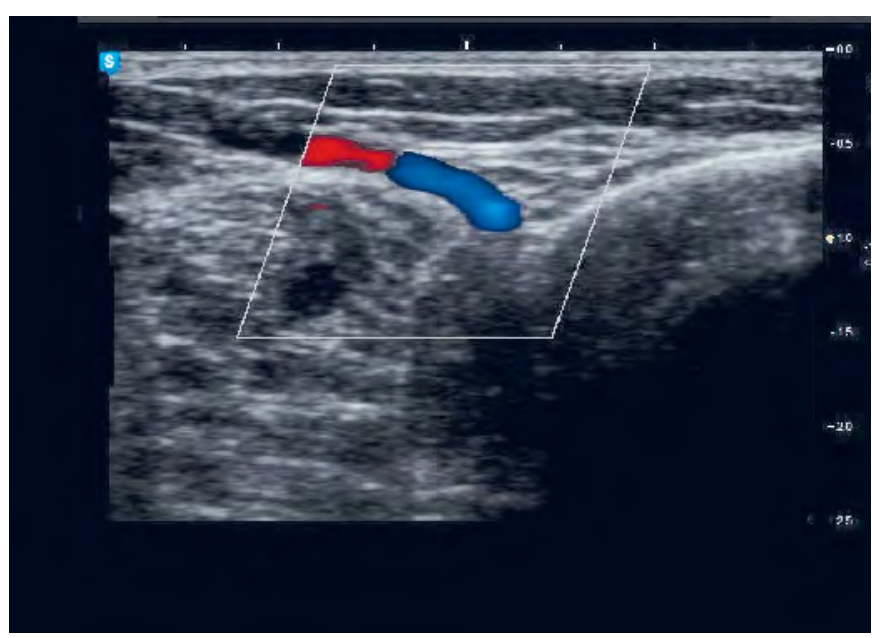

Abb. 8

Prätibiale Perforansvene ohne pathologischen Reflux

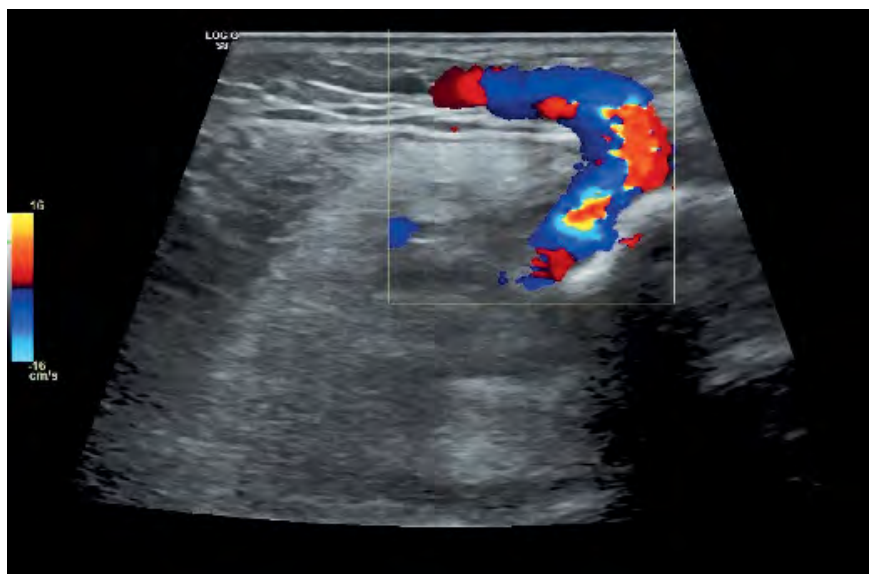

Abb. 9

Insuffiziente Perforansvene, der Fluss nach Dekompression ist zur Oberfläche gerichtet

Je weiter distal am Bein eine Perforansvene liegt, desto unwahrscheinliche ist es, dass sie der Rezirkulation als Insuffizienzpunkt dient. Ausnahme hierzu bilden die sekundären Varizen nach Traumata der Wade. Häufiger liegen einzige refluxive Muskelvenen nach Traumata vor. Diese füllen dann Seitenastvarizen und bilden deren proximalen Insuffizienzpunkt. (4)

Nicht selten findet man die insuffizienten Muskelvenen als Ursprung für subkutane retikuläre Varizen. Meistens handelt es sich um posttraumatische Varizen.

Es ist sehr selten, dass eine gedehnte Perforansvene eine bereits refluxive Vene zusätzlich füllt, kann jedoch bei einer sekundären Varikose vorkommen. Ist die Vene oberhalb der Perforansvene bereits refluxiv, muss man akkurat die Flusskurven in der V. saphena und der Perforansvene vergleichen, bevor man diese als therapiebedürftige insuffiziente Perforans bezeich- net. Deren Flusskurven muss überwiegend auswärts gerichtet sein und die Flusskurven der distalen Vene ein größeres Refluxvolumen führen, als in deren proximalen Anteil. Diese Untersuchung ist nur im PW Modus möglich.

Die Perforansvenen können als Drainage dienen. Am häufigsten sind es Venen der Wade, insbesondere die Cockett-Gruppe und die paratibialen Perforansvenen $(\triangleright$ Abb. 10).

\section{Phlebographie}

Die früher häufig genutzte Phlebographie mit Kontrastmittel hat angesichts der Möglichkeiten der Duplexsonographie an Bedeutung in der Diagnostik der Perforansinsuffizienz verloren. Die Indikation zur phlebographische Diagnostik ist mit einer konkreten Fragestellung nach stattgefunde-

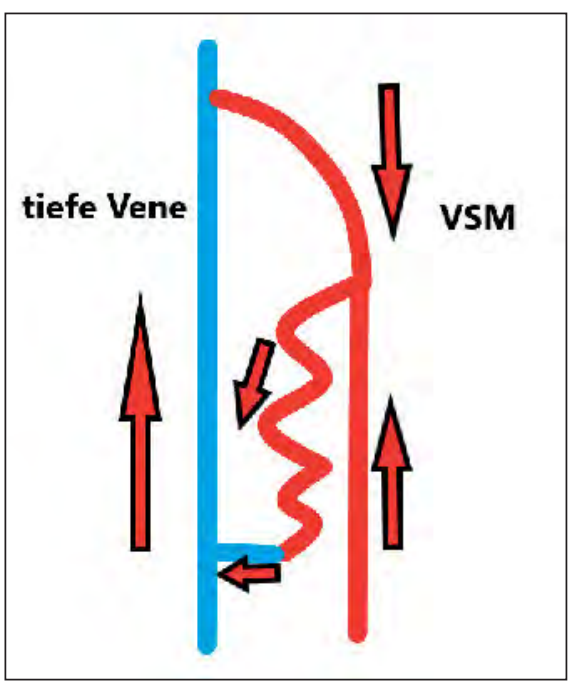

Abb. 10 Insuffizienz der V. saphena magna mit konjungierende Seitenastvarikose, Drainage über die Perforansvene (Flussrichtung mit Pfeilen angezeichnet).

ner duplexsonographischen Untersuchung zu stellen. Sie kann in seltenen Fällen sinnvoll sein bei mehrfach voroperierten Beine, bei stattgehabten frischen Thrombose oder extremen Adipositas ( $\triangleright$ Abb. 11 und 12).

\section{Ulcus cruris venosum}

Es besteht eine Korrelation zwischen der Anzahl insuffizienter Perforanten und dem Schweregrad der CVI, bzw. der Entstehung eines Ulcus cruris venosum. Als alleinige Ursache für das venöse Ulkus ist die Perforansinsuffizienz selten, mit etwas größerer Bedeutung der Cockett Perforans. Phlebodynamometrischen Studien zufolge steigt das Risiko für die Entwicklung eines venösen Ulkus mit der Schwere der hämodynamischen Dekompensation. Eine dauerhafte Ulkusheilung ist nur bei einer weitgehenden Normalisierung der ambulatorischen venösen Hypertonie zu erwarten. Bei alleiniger Insuffizienz des oberflächlichen Venensystems und begleitender Perforans-Insuffizienz genügt meist ausschließlich die Ausschaltung der epifaszialen Refluxkomponente. Im Gegensatz dazu persistiert die venöse Hypertonie nach Sanierung der epifaszialen Insuffizienzen auch nach zusätzlicher Ausschaltung insuffizienter Perforantes, wenn eine gleichzeitige irreversible 
Schädigung des tiefen Venensystems vorliegt $(5,6)$.

Einige Studien zeigen, dass nach Ausschaltung des epifaszialen Refluxes (Kross-

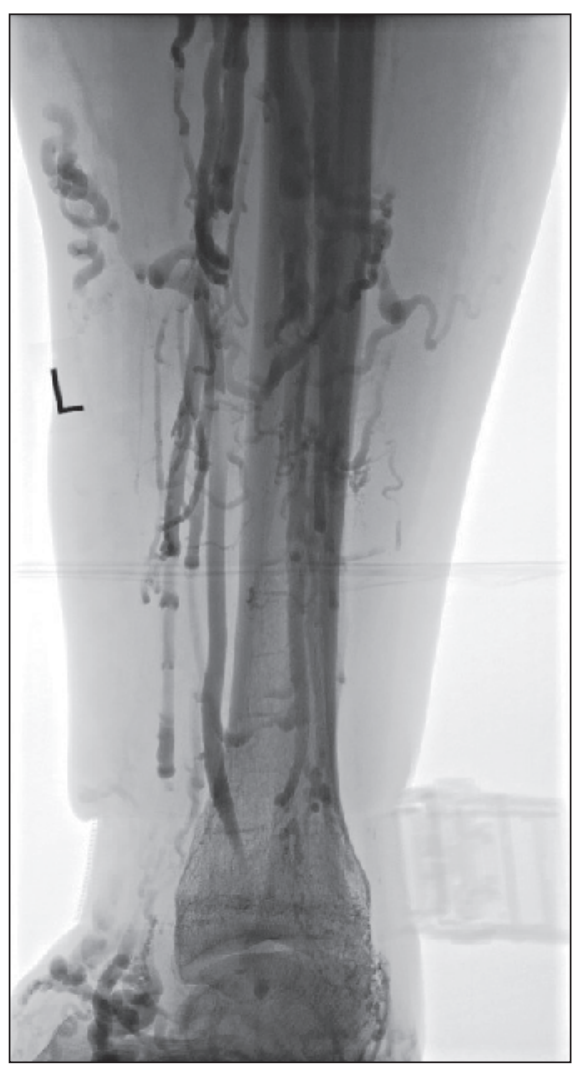

Abb. 11 Phlebologischer Nachweis einer Perforansinsuffizienz Cockett III bei bestehender Rezidivvarikosis, schwerem sekundären Lymphödem und venösen Ulzerationen nach Crossektomie und Stripping

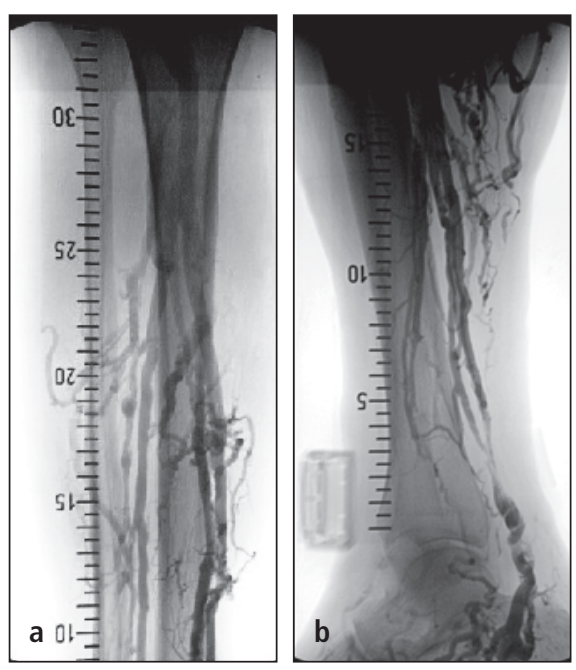

Abb. 12 Stamminsuffizienz der VSM Hach II mit insuffizient imponierenden Dodd-Perforansvenen ( $a$ und $b$ ) ektomie und Stripping) die Anzahl präoperativ bestehender Perforansveneninsuffizienzen abnimmt $(7,8)(\triangleright$ Abb. 13 und 14).

Abb. 13

Chronische venöse Ulzera bei einer Perforansinsuffizienz Cockett III
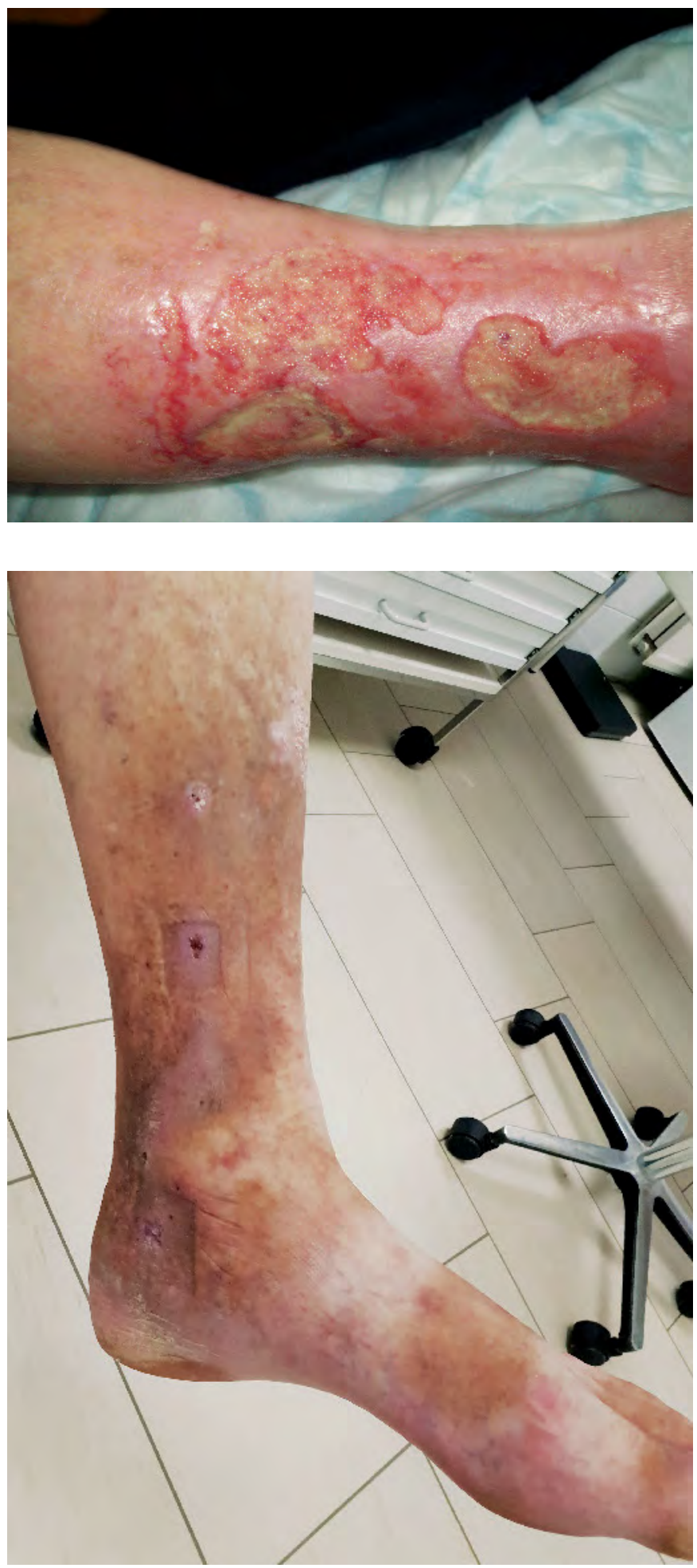

\section{Therapiemethoden}

Bei klinisch und/oder hämodynamisch relevanter Perforansinsuffizienz ist die Ausschaltung der insuffizienten Perforansvenen zu erwägen (9-11). Der Nutzen eines

Abb. 14

Nach Sklerosierungsbehandlung abheilende Ulcus cruris venosum bei Perforansinsuffizienz 


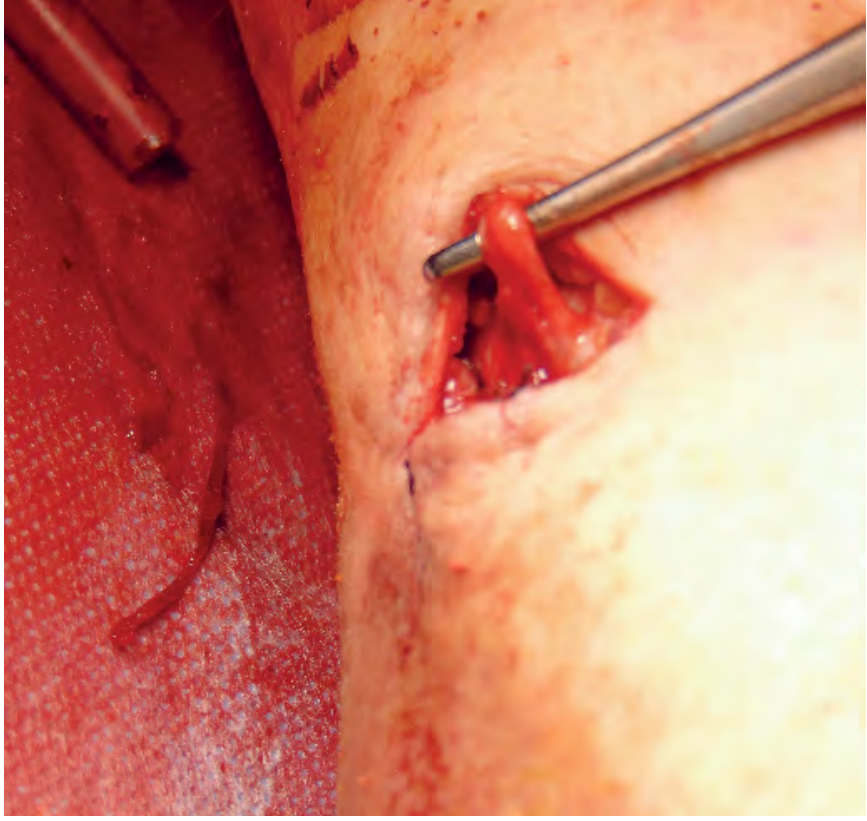

Abb. 15

Operative Freilegung und Ligatur einer $\mathrm{V}$. perforans

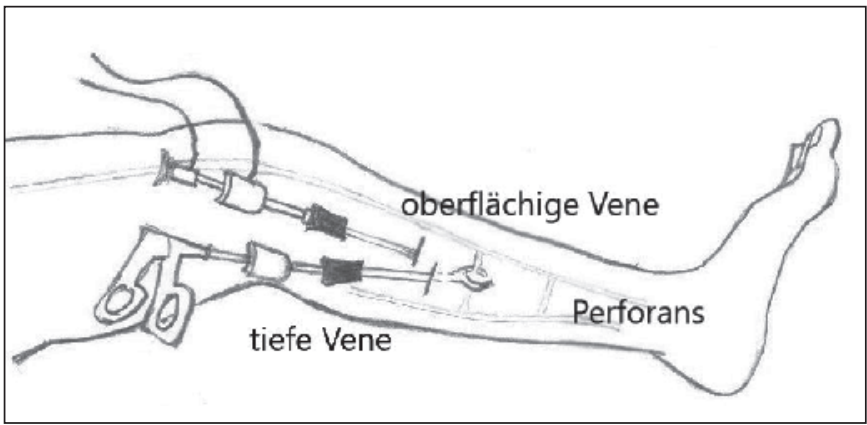

Abb. 16

Endoskopische Perforansligatur (schematische Darstellung)

routinemäßigen ausschalten von Perforansvenen bei der unkomplizierten, rein epifaszialen Varikose ist nicht belegt (11-13). Bei gesunden Hautverhältnissen ist die operative Unterbindung der Perforansvenen derzeit das komplikationsärmste Verfahren (11) ( Abb. 15 und 16).

Die Freilegung der Perforansvenen nach Linton (16), ohne Rücksicht auf ihre pathophysiologische Bedeutung und die Hautsituation, wird nicht mehr empfohlen (11).

In der Literatur sind Fälle der endovenösen Therapie bei der Perforansinsuffizienz beschrieben worden $(17,18)$. Es besteht jedoch noch keine Therapieempfehlung der endovenösen Laser oder Radiofrequenzbehandlung der Perforanten (11).

Die Duplexgesteuerte Schaumsklerosierung der Perforansvenen zeigt gute Studienergebnisse und ist bei schlechtem Haut- zustand als mögliche Behandlung vorzuziehen $(19,20)$.

\section{Interessenkonflikt}

Nach Angaben der Autoren bestehen keine Interessenkonflikte.

\section{Ethische Richtlinien}

Für das Manuskript wurden keine Studien an Menschen oder Tieren durchgeführt.

\section{Schlussfolgerung}

Perforansvenen sind eine Besonderheit der unteren Extremität und bringen eine Herausforderung bei der Diagnostik und Therapie der chronischen venösen Insuffizienz mit sich. Die pathophysiologische Bedeu- tung der entdeckten Perforansvenen kann durch sorgfältige Duplexdiagnostik überprüft werden. Die Perforansvenen können einen Insuffizienzpunkt bilden, häufiger dienen sie aber als Drainage für einer venösen Rezirkulation.

\section{Literatur}

1. Gloviczki P. Handbook of venous disorders.. 3rd ed. London: Edward Arnold 2009.

2. Van Limborgh J, Hage E. Anatomical features of those perforating veins of the legwhich frequently or infrequently become incompetent. In May R, Partsch H, Staubesand J. Perforating veins. München: Urban\&Schwarzenberg 1981, 49-59.

3. Caggiati A, Bergan J, Gloviczki P, Jantet G, Wendell-Smith C, Partsch H. Nomenclature of the veins of the lower limbs: an international interdisciplinary consensus statement. J Vasc Surg 2002; 36: 416-422.

4. Mendoza E. Duplexsonographie der oberflächlichen Beinvenen. 2nd ed. Springer-Verlag GmbH 2013.

5. Klein-Weigel P, Biedermann H, Fraedrich G. The role of perforating vein dissection in the treatment of venous ulceration - myth and evidence. Vasa 2002

6. Recek C, Karisch E, Gruber J. Veränderungen der Perforansvenen und tiefen Unterschenkelvenen nach Beseitigung des Saphena-Refluxes. Phlebologie 2000; 29(02): 37-40.

7. Blomgren L, Johansson G, Dahlberg-Akerman A, Thermaenius P, Bergqvist D. Changes in superficial and perforating vein reflux after varicose vein surgery. J Vasc Surg 2005; 42(2): 315-320.

8. Gohel M, Barwell J, Earnshaw J, Heather B, Mitchell D, Whyman M, et al. Randomized clinical trial of compression plus surgery versus compression alone in chronic venous ulceration (ESCHAR study) - haemodynamic and anatomical changes. Br J Surg 2005; 92(3): 291-297.

9. Fitridge R, Dunlop C, Raptis S, Thompson M, Leppard P, Quigley F. A prospective randomized trial evaluating the haemodynamic role of incompetent calf perforating veins. Aust N Z J Surg 1999; 69: 214-216.

10. Hach W, Hach-Wunderle V, Nestle W. Die Insuffizienz der Cockett-Vv.-perforantes und die operative Behandlung. Gefäßchirurgie 2000; 5: $130-137$.

11. Kluess H, Noppeney T, Breu F, Ehresmann U, Gerlach H, Hermanns H, et al. Leitlinie zur Diagnostik und Therapie der Krampfadererkrankung. Phlebologie 2004

12. Bassi G. Klinische Bedeutung insuffizienter Vv. Perforantes. In May R, Partsch H, Staubesand J. Venae perforantes. München: Urban\&Schwarzenberg 1981: 110-113.

13. Bjordal R. Die Zirkulation in insuffizienten Vv. Perforantes der Wade bei venösen Störungen. In May R, Partsch H, Staubesand J. Venae perforantes. München: Urban\&Schwarzenberg 1981: 71-88. 
14. Hauer G. Operationstechnik der endoskopischen subfascialen Discision der Perforansvenen. Chirurg 1987; 58: 172-175.

15. Hauer G, Nasralla F, Wisser I, Schneidemann G. Zur endoskopischen Perforansdissektion und Fasziotomie. Gefäßchirurgie 1997; 2: 222-226.

16. Linton R. The post-thrombotic ulceration of the lower extremity: its etiology and surgical treatment. Ann Surg 1953; 138: 415-432.

17. Perälä J, Rautio T, Biancorie F, Ohtonen P, Wiik H, Heikkinen $\mathrm{T}$ et al. Radiofrequency endovenous obliteration versus stripping of the long saphenous vein in the management of primary varicose veins; 3 -year outcome of a randomized study. Ann Vasc Surg 2005; 19: 1-4.

18. Proebstle T, Herdemann S. Early results and feasibility of incompetent perforator vein ablation by endovenous laser treatment. Dermatol Surg 2007; 33: $162-168$.

19. Kishore R, Sankar T, Anandi A, Nedunchezhian S. A Prospective Study in Comparison of Ambulatory Phlebectomy and Duplex Guided Foam Scle- rotherapy in the Management of Varicosities with Isolated Perforator Incompetence. Indian J Surg 2016; 78(5): 356-363.

20. Dillavou E, Harlander-Locke M, Labropoulos N, Elias S, Ozsvath K. Current state of the treatment of perforating veins. J Vasc Surg Venous Lymphat Disord 2016; 4(1): 131-135. 